\title{
REVISÃO INTEGRATIVA DA LITERATURA SOBRE VIOLÊNCIA DOMÉSTICA E O DESENVOLVIMENTO DE DEPRESSÃO PÓS-PARTO
}

\begin{abstract}
Rafaela Joy Falcão', ORCID ID 0000-0002-5488-5049; Maria Vitória da Silva Paula Cirilo', ORCID ID 0000-00034869-8279; Anna Karolyna da Silva Queiroz de Sá 1, ORCID ID 0000-0003-1663-2566; Brunna Hatsune Kihara Rocha1, ORCID ID 0000-0001-5614-4247; Anna Maria Andrade Barbosa1', ORCID ID 0000-0001-5614-4247; Luiz Henrique Fernandes Musmanno², ORCID ID 0000-0003-4837-4864
\end{abstract}

\section{FILIAÇÃO}

(1) Pontifícia Universidade Católica de Goiás, Escola de Ciência Médicas, Farmacêuticas e Biomédicas, Discente de Medicina

(2) Pontifícia Universidade Católica de Goiás, Escola de Ciência Médicas, Farmacêuticas e Biomédicas, Mestre, Professor do curso de Medicina.

\section{AUTOR CORRESPONDENTE}

Rafaela Joy Falcão, rafajoyfalcao@gmail.com, Pontifícia Universidade Católica de Goiás, Escola de Ciência Médicas, Farmacêuticas e Biomédicas.

\section{MENSAGENS-CHAVE}

A depressão pós-parto é um reconhecido problema de saúde pública, que acarreta significativa morbimortalidade materna e infantil.

Dentre as principais consequências da depressão pós-parto, há a diminuição do vínculo mãe-filho e o suicídio materno.

As mulheres vítimas de violência doméstica possuem um maior risco de desenvolver a DPP pois a violência é um evento traumático.

A violência psicológica foi descrita como a mais prevalente, embora a violência física seja a mais relacionada à $D P P$.

Para reduzir o risco de DPP, é crucial o rastreio da violência doméstica e o correto encaminhamento pelos profissionais de saúde.

\section{RESUMO}

INTRODUÇÃO: A violência doméstica (VD) é definida por "...qualquer ação ou omissão baseada no gênero que lhe cause morte, lesão, sofrimento físico, sexual ou psicológico e dano moral ou patrimonial". Esse tipo de agressão, que repercute em vários aspectos da vida da mulher, tem sido relacionada à depressão pós-parto. METODOLOGIA: Trata-se de uma revisão integrativa de literatura cujo objetivo foi verificar a relação entre violência doméstica e depressão pós-parto, por meio da estratégia PICO. A busca foi realizada em abril de 2021 nas fontes de dados eletrônicas MEDLINE/PubMed, Sciencedirect e Google acadêmico usando os descritores "domestic violence" AND "postpartum depression" e o filtro de 5 anos. Ao final, 22 artigos compuseram a presente revisão (12 PubMed, 4 Sciencedirect, 6 Google acadêmico). RESULTADOS: Todos os artigos revisados apresentaram associação entre violência doméstica e depressão pós-parto (DPP). Além disso, foi observada, também, relação com ideações suicidas. A violência psicológica foi descrita como a mais prevalente, embora a violência física seja a mais relacionada à DPP. A prevalência de casos de violência encontrada foi de $4,9 \%$ a $67,4 \%$ enquanto a prevalência de DPP ficou entre 4,4\% e 41,5\%. DISCUSSÃO: A DPP é reconhecidamente um problema de saúde pública, com significativa morbimortalidade materna e infantil, com prevalência entre 10\%, em países de alta renda, e $60 \%$ na população da África Subsaariana. A prevalência de DPP e violência doméstica encontradas corresponde ao encontrado na literatura mundial. Dentre as principais consequências da DPP, destacam-se a diminuição do cuidado com o recém-nascido e o suicídio materno. CONCLUSÃO: O presente artigo conclui que é necessária uma maior sensibilidade pelos profissionais de saúde no rastreamento das violências domésticas para que as mulheres recebam os devidos cuidados e apoio visando 0 estabelecimento de estratégias intersetoriais que permitam que essas mulheres saiam das situações abusivas. 


\section{ABSTRACT}

INTRODUCTION: Domestic violence (DV) is defined as "....any action or omission based on gender that causes death, injury, physical, sexual or psychological suffering and moral or property damage". This type of aggression, which affects various aspects of a woman's life, has been linked to postpartum depression. METHODOLOGY: This is an integrative literature review whose objective was to verify the relationship between domestic violence and postpartum depression, through the PICo strategy. The search was performed in April 2021 in the electronic data sources MEDLINE/PubMed, Sciencedirect and Academic Google using the descriptors "domestic violence" AND "postpartum depression" and the 5-year filter. At the end, 22 articles composed this review (12 PubMed, 4 Sciencedirect, 6 Google academic). RESULTS: An association was observed between intimate partner violence and postpartum depression (PPD) in all reviewed articles. In addition, a relationship with suicidal ideations was also perceived. Psychological violence was described as the most prevalent, although physical violence is the most related to PPD. The prevalence of cases of violence found was $4.9 \%$ to $67.4 \%$, while the prevalence of PPD was between $4.4 \%$ and $41.5 \%$. DISCUSSION: PPD is recognized as a public health problem, with significant maternal and child morbidity and mortality, with a prevalence between $10 \%$ in high-income countries and $60 \%$ in the population of sub-Saharan Africa. The prevalence of PPD and domestic violence found corresponds to that found in world literature. Among the main consequences of PPD, the decrease in care for the newborn and maternal suicide stand out. CONCLUSION: This article concludes that greater sensitivity is needed by health professionals in tracking -domestic violence for women to receive proper care and support in order to establish intersectoral strategies that allow these women to get out of abusive situations.

\section{KEYWORDS: Domestic Violence; Postpartum Depression; Pregnant Women}

\section{INTRODUÇÃO}

A violência doméstica (VD) contra a mulher repercute em vários aspectos da sua vida, como trabalho, relações sociais e na saúde, tanto física quanto mental. É definida pelo artigo $5^{\circ}$ da Lei $n^{\circ}$ 11.340/2006, conhecida como Lei Maria da Penha, como qualquer ação ou omissão baseada no gênero que the cause morte, lesão, sofrimento físico, sexual ou psicológico e dano moral ou patrimonial (1). Essa lei rege os mecanismos para redução da violência doméstica e familiar contra a mulher e define os tipos de violência, a saber: física, patrimonial, sexual, moral e psicológica.

A violência física é definida como a ação de ferir e/ou causar danos e lesões ao corpo. Já a violência patrimonial refere-se à destruição de bens materiais e objetos. A violência sexual acontece quando o agressor obriga a vítima a presenciar, manter ou a participar de relação sexual não desejada. A violência moral constitui calúnia e difamação contra a outra pessoa, enquanto a violência psicológica ou emocional, constitui a mais silenciosa, sendo caracterizada por qualquer conduta que resulte em dano emocional, podendo haver inúmeras consequências como a depressão pós-parto $(1,2)$.

A depressão pós-parto é definida como um transtorno do humor que se inicia, geralmente, nas primeiras quatro semanas após o parto e pode ser de intensidade variável, chegando a ser considerada uma desordem psicótica. A depressão maior ocorre com a presença do humor depressivo, ou diminuição ou perda do interesse nas atividades anteriormente agradáveis, associada a pelo menos três dos sintomas: insônia ou sono excessivo, alteração significativa de peso ou do apetite, fadiga, sentimentos de desvalia ou culpa, agitação ou lentidão psicomotora, perda de concentração e ideias de morte ou suicídio (3).

A violência doméstica tem sido relacionada à depressão, muitas vezes, apontada como uma de suas causas, devido ao fato de repercutir sobre inúmeros aspectos da vida da mulher. De acordo com a Organização Mundial da Saúde, em 2013, 30\% das mulheres no mundo já foram vítimas de violência doméstica (4).

Enquanto por um lado a gravidez pode ser fator protetor contra a VD, podendo ocorrer a diminuição ou a interrupção de episódios abusivos; por outro lado ela pode determinar o início de agressões ou aumento de sua frequência e gravidade. Em mais de $90 \%$ dos casos registrados, os maus tratos já estavam presentes, sendo que a história de VD pode ser o principal preditor de agressões durante a gravidez (3). Entretanto, ainda que seja observável a relação entre essas duas problemáticas, estudos que as relacionem ainda estão em falta.

Este trabalho tem por objetivo revisar a literatura visando identificar, entre os estudos, a relação existente entre violência doméstica e depressão pós-parto, atestando se há algum impacto negativo nessa correlação.

\section{METODOLOGIA}

Trata-se de uma revisão integrativa da literatura. fundamentada na estratégia PICo (P-população: mulheres; IInteresse: depressão pós-parto; Co- Contexto: mulheres vítimas de violência doméstica), o que resultou na seguinte questão norteadora: qual a relação entre o desenvolvimento da depressão pós-parto em mulheres vítima de violência doméstica? (5)

A busca da literatura utilizada para compor a revisão foi feita por um pesquisador de maneira independente e realizada entre 18/04/21 e 29/04/21 nas fontes de dados eletrônicas MEDLINE/PubMed (Medical Literature Analysis and Retrieval System Online), Sciencedirect e Google acadêmico utilizando a combinação dos descritores: "domestic violence" AND "postpartum depression", utilizou-se filtro 5 anos para a busca.

Foram incluídos todos os estudos de coorte, transversal, multicêntricos e revisões sistemáticas com metanálise encontrados nas bases de dados. Os critérios de inclusão foram: artigos publicados em português, inglês e espanhol, nos últimos 5 anos, que atendiam a temática do presente artigo e 
com disponibilidade de texto completo em suporte eletrônico. Foram excluídos os estudos duplicados. Já os critérios de exclusão foram os que não foram realizados com seres humanos e àqueles trabalhos que não eram compatíveis com os critérios ou temática propostos para esta revisão: artigos que tratavam de outros tipos de violência que não seja a doméstica e os que a relação causal analisada divergia do presente estudo.

Inicialmente, havia 267 artigos disponíveis, dos quais foram selecionados 23 (13 PubMed, 4 Sciencedirect, 6 Google acadêmico) após a leitura inicial dos títulos e consequente exclusão daqueles cuja temática não era pertinente ao objetivo desse estudo e, portanto, não preenchiam os critérios de inclusão/exclusão acima descritos. Ao final 22 compuseram a presente revisão, sendo excluído um artigo do PubMed que apresentava apenas o projeto de pesquisa (Figura 1).

Além disso, foram extraídas as seguintes características dos estudos: título, autores, desenho de estudo e principais achados (Tabela 1).

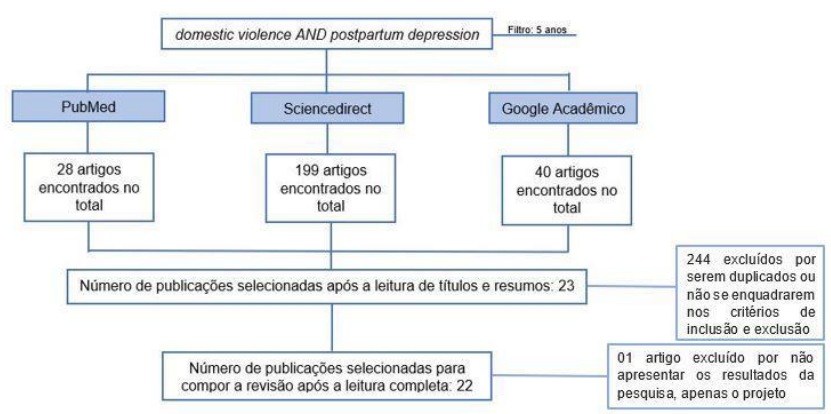

Fluxograma das seleção e exclusão dos estudos com as bases de dados e seus respectivos resultados.

Fonte: Própria, 2021

\section{RESULTADOS}

Dos 22 estudos selecionados, 11 são estudos transversais $(6,7,9,11,12,13,14,15,16,20,21)$ e 5 são longitudinais $(8,10,17,18,25)$. Entre os estudos transversais e longitudinais, a Escala de Depressão Pós-parto de Edimburgo (EPDS) foi a mais usada para estimar a prevalência da DPP. As ferramentas utilizadas pelos três artigos que não usaram a EPDS foram: a escala PHQ-9 (6), a versão SRQ-20 validada no Brasil (7) e, no último caso, foi utilizada uma amostra de mulheres internadas já diagnosticadas com DPP, sem necessidade de uso de uma ferramenta para esse fim diagnóstico (8).

Já em relação a prevalência da violência doméstica, as ferramentas usadas foram mais heterogêneas, muitas vezes sendo usadas apenas perguntas separadas sobre violência física, sexual e psicológica ou utilizando-se dos Questionários de Pesquisa Demográfica ou Saúde da Mulher da OMS $(6,9,10,11,12)$. Deve-se frisar que todos os artigos encontraram relação significativa entre os diversos modos de violência e a DPP.

Entre os estudos transversais, a prevalência de sintomas de DPP variou entre 4,4\% (12) e $41,5 \%$ (13). A prevalência de violência também apresentou grandes variações. O estudo de Islam et al. (2017) apresentou os maiores níveis de violência por parceiro íntimo (VPI), com a VPI física chegando a 52,8\% antes da gravidez, a VPI psicológica a $67,4 \%$ e a VPI sexual a $21,1 \%$, decaindo a partir da gravidez e do parto.

No entanto, em Ahmad et al. (2018), a prevalência de VPI foi de $4,9 \%$.

Em relação ao maior risco das vítimas de VPI desenvolverem DPP, quando comparadas às mulheres não vítimas, esse número variou entre 2,3 (12) e 6,74 (13). A maioria dos estudos relatou a VPI emocional como a forma de violência mais prevalente $(11,12,14,15)$. Todavia, ela foi apontada como a mais relacionada a DPP em apenas um estudo 16 ao contrário da violência física, apontada como a mais significativa em três $(9,11,14)$ e menos prevalente em dois artigos $(11,14)$, chegando a aumentar o risco de DPP em sete vezes (14).

Um dado interessante trazido por Manongi et al. (2020) foi que a exposição à violência emocional só se mostrou significativa numa amostra de mulheres com HIV/AIDS, aumentando as chances de DPP em 3 vezes neste grupo. Em Gibbs et al. (2017), foi observada sobreposição entre VPI, sintomas depressivos e qualquer limitação/incapacidade funcional, onde $72 \%$ das mulheres que sofreram com VPI também tinham sintomas depressivos e/ou limitação/incapacidade funcional. Além disso, Tabb et al. (2018) trouxeram um dos grandes impactos da DPP: o suicídio materno. Enquanto a prevalência de DPP foi de $28 \%$ na amostra geral, $89 \%$ das mulheres que relataram ideações suicidas possuíam DPP. Nessa amostra, a prevalência de VPI 6-9 meses após o parto foi de $21,2 \%$, chegando a $31,1 \%$ em 12 meses após o parto.

Nos estudos longitudinais, a prevalência de violência variou entre $11,07 \%$ (17) até 44\% (8). Em Rogathi et al. (2017), a violência física ou violência sexual aumentaram em duas vezes a probabilidade de desenvolver DPP contra um aumento de 3,5 vezes em Finnbogadóttir et al. (2016). Ademais, em Rogathi et al. (2017) a prevalência de violência emocional chegou a $22,3 \%$, a sexual a $15,7 \%$ e a física a $6,2 \%$, o que contrasta com as prevalências encontradas por Hou et al. (2020) de violência física de 4,55\%, de violência psicológica de $9,23 \%$ e de sexual de $2,34 \%$.

Destaca-se o trabalho de Rogathi et al. (2017) que apresenta que no grupo de mulheres sem histórico de depressão, a violência emocional apresentou risco duas vezes maior de apresentar DPP, assim como as violências física e emocional, embora isso não tenha sido visto nas mulheres com histórico de depressão. Adicionalmente, as mulheres de 18 a 24 anos apresentaram quatro vezes mais chance de desenvolver sinais de DPP, quando expostas a violência física, e o dobro das chances em mulheres acima de 24 anos.

Por último, num estudo realizado por Reilly (2018), foi avaliado o papel dos profissionais de saúde no rastreamento de casos de violência em serviços de pré-natal e pós-parto. Os fatores identificados para falta de rastreamento de violência foram: a falta de reconhecimento de que deveriam desempenhar essa função; a falta de políticas de triagem de violência doméstica; a falta de tempo, recursos e confiança para realizar o rastreamento e encaminhamento das mulheres quando detectada violência doméstica. Assim, tais fatores impedem que os profissionais possam, de fato, ajudar efetivamente as mulheres a saírem de situações abusivas e receberem cuidados adequados (19).

\section{DISCUSSÃO}


Tabela 01

\begin{tabular}{|c|c|c|c|}
\hline Autor & Título & Características metodológicas & Principais achados \\
\hline $\begin{array}{l}\text { (6) Gibbs A, Carpenter } \\
\text { B, Crankshaw T, } \\
\text { Hannass-Hancock J, } \\
\text { Smit J, Tomlinson M, } \\
\text { et al. }\end{array}$ & $\begin{array}{l}\text { Prevalence and factors } \\
\text { associated with recent } \\
\text { intimate partner violence and } \\
\text { relationships between } \\
\text { disability and depression in } \\
\text { postpartum women in one } \\
\text { clinic in eThekwini } \\
\text { Municipality, South Africa. }\end{array}$ & $\begin{array}{l}\text { Estudo transversal entre } 275 \text { mulheres que se apresentaram } \\
\text { para cuidados pós-parto numa clínica no municipio de } \\
\text { eThekwini, África do Sul. A DPP foi avaliada pela escala } \\
\text { PHQ-9 e a VPI através da Pesquisa de Violência contra as } \\
\text { Mulheres da Organização Mundial de Saúde (OMS) } \\
\text { modificada para a África do Sul. Foi usado o } 12 \text { item } \\
\text { WHODAS 2.0, que mede as limitações funcionais (cognição, } \\
\text { mobilidade, autocuidado, convivência, participação e } \\
\text { gerenciamento de atividades de vida). }\end{array}$ & $\begin{array}{l}\text { Foi visto sobreposição entre VPI, sintomas depressivos e } \\
\text { qualquer limitação funcional: } 72 \% \text { das que sofreram com VPI } \\
\text { também tinham sintomas depressivos e / ou incapacidade } \\
\text { funcional. A relação entre a DPPe a VPI é provavelmente } \\
\text { bidirecional, com a depressão como causa e consequência da } \\
\text { VPI. Não houve relação entre VPI e qualquer fator específico } \\
\text { de gravidez, o que sugere que a VPI não depende de fatores } \\
\text { da gravidez, mas sim fatores sociais mais amplos relacionados } \\
\text { a vulnerabilidade. }\end{array}$ \\
\hline $\begin{array}{l}\text { (7) Tabb KM, Huang } \\
\mathrm{H} \text {, Valdovinos M, Toor } \\
\mathrm{R} \text {, Ostler T, } \\
\text { Vanderwater } \mathrm{E} \text {, et al. }\end{array}$ & $\begin{array}{l}\text { Intimate Partner Violence Is } \\
\text { Associated with Suicidality } \\
\text { among Low-Income } \\
\text { Postpartum Women. }\end{array}$ & $\begin{array}{l}\text { Estudo transversal entre } 701 \text { puérperas recrutados em } \\
\text { clínicas de atenção primária em São Paulo. As ideações } \\
\text { suicídas pós-parto foram avaliadas usando o cronograma de } \\
\text { entrevista clínica revisado. A DPP foi avaliada pela versão } \\
\text { SRQ-20 validada no Brasil. A VPI foi avaliada por meio de um } \\
\text { questionário estruturado previamente validado em } \\
\text { populações brasileiras, por meio da variável dicotômica } \\
\text { construída de violência pós-parto e inclui violência } \\
\text { psicológica, fisica e emocional. }\end{array}$ & $\begin{array}{l}\text { A prevalência de ideações suicídas pós-parto foi de } 4 \% \text {. Dentre } \\
\text { essas mulheres com ideações suicidas, } 70 \% \text { relataram VPI } \\
\text { durante o periodo pós-parto. Desse modo, mulheres no pós- } \\
\text { parto expostas à violência apresentam risco três vezes maior } \\
\text { de ter pensamentos suicidas. }\end{array}$ \\
\hline $\begin{array}{l}\text { (8) Ghajati B, Bram N, } \\
\text { Tajmout A, Ben } \\
\text { Romdhane I, Homri W, } \\
\text { Labbane R. }\end{array}$ & $\begin{array}{l}\text { Domestic Violence and } \\
\text { Postpartum Depression: a } \\
\text { Study About } 25 \text { Tunisian } \\
\text { Women. }\end{array}$ & $\begin{array}{l}\text { Estudo retrospectivo, descritivo e analítico entre } 25 \text { mulheres } \\
\text { que sofrem de DPP internadas no departamento de } \\
\text { psiquiatria. Pacientes foram avaliadas em relação à violência } \\
\text { doméstica pela Woman Abuse Screening Tool (WAST). }\end{array}$ & $\begin{array}{l}\text { Dentre as mulheres da amostra, } 44 \% \text { relataram abuso, } \\
\text { especialmente emocional. Três pacientes cometeram suicídio. } \\
\text { Conclui-se que a violência doméstica causa uma gama de } \\
\text { consequências psicológicas e físicas. Ademais, profissionais de } \\
\text { saúde mental devem ser capazes de, precocemente, identificar } \\
\text { vítimas de abuso para prevenir que tais complicações ocorram. }\end{array}$ \\
\hline $\begin{array}{l}\text { (9) Islam MJ, Broidy L, } \\
\text { Baird K, Mazerolle P. }\end{array}$ & $\begin{array}{l}\text { Intimate partner violence } \\
\text { around the time of pregnancy } \\
\text { and postpartum depression: } \\
\text { The experience of women of } \\
\text { Bangladesh. }\end{array}$ & $\begin{array}{l}\text { Estudo transversal entre } 426 \text { novas mães, em Bangladesh, } \\
\text { entre } 15-49 \text { anos, que estavam nos primeiros seis meses } \\
\text { após o parto. ADPP foi avaliada por meio da EPDS e a } \\
\text { violência doméstica pelo módulo de violência doméstica do } \\
\text { Questionário de Pesquisa Demográfica de Saúde da OMS. }\end{array}$ & $\begin{array}{l}\text { Aproximadamente } 35,2 \% \text { das mulheres tiveram DPP nos } \\
\text { primeiros seis meses após o parto. A exposição à VPI } \\
\text { aumentou significativamente as chances de DPP. A associação } \\
\text { é particularmente forte para violência fisica durante todos os } \\
\text { periodos, com um OR ajustado de } 1.79 \text {, e VPI psicológica } \\
\text { durante a gravidez, com um OR ajustado de } 6.92 \text {. As } \\
\text { pontuações médias de EPDS foram mais altas para mulheres } \\
\text { que tinham experimentado VPI física, seguida de sexual e } \\
\text { psicológica. }\end{array}$ \\
\hline $\begin{array}{l}\text { (10) Rogathi JJ, } \\
\text { Manongi R, Mushi D, } \\
\text { Rasch V, Sigalla GN, } \\
\text { Gammeltoft T, et al. }\end{array}$ & $\begin{array}{l}\text { Postpartum depression among } \\
\text { women who have experienced } \\
\text { intimate partner violence: A } \\
\text { prospective cohort study at } \\
\text { Moshi, Tanzania. }\end{array}$ & $\begin{array}{l}\text { Estudo de coorte prospectivo entre } 1.013 \text { mulheres grávidas } \\
\text { com menos de } 24 \text { semanas de gestação atendidas em } \\
\text { clinicas pré-natais na região de Kilimanjaro, Tanzânia.A DPP } \\
\text { foi avaliada pela EPDS e a violência doméstica por intermédio } \\
\text { de questões do Estudo Multi-Países sobre Saúde da Mulher e } \\
\text { Violência Doméstica. Foram entrevistadas em } 4 \text { momentos, } \\
\text { sendo } 3 \text { durante o pré natal e a avaliação da DPP usando } \\
\text { EPDS foi repetida } 40 \text { dias após o parto. }\end{array}$ & $\begin{array}{l}\text { Uma em cada três mulheres grávidas na população do estudo } \\
\text { foi exposta à VPI durante a gravidez e esses eventos foram } \\
\text { fortemente associados ao desenvolvimento de DPP, com um } \\
\text { risco cerca de } 3 \text { vezes maior do que as não expostas. Mulheres } \\
\text { mais jovens e mulheres sem histórico anterior de depressão } \\
\text { apresentaram maior risco de desenvolver DPP quando } \\
\text { expostas à violência. }\end{array}$ \\
\hline $\begin{array}{l}\text { (11) Manongi R, } \\
\text { Rogathi J, Sigalla G, } \\
\text { Mushi D, Rasch V, } \\
\text { Gammeltoft T, et al. }\end{array}$ & $\begin{array}{l}\text { The Association Between } \\
\text { Intimate Partner Violence and } \\
\text { Signs of Depression During } \\
\text { Pregnancy in Kilimanjaro } \\
\text { Region, Northern Tanzania. }\end{array}$ & $\begin{array}{l}\text { Estudo transversal entre } 1.116 \text { gestantes atendidas no } \\
\text { municipio de Moshi, Tanzânia. ADPP foi avaliada por meio } \\
\text { da EPDS e a exposição à VPI foi avaliada pelo questionário } \\
\text { estruturado da OMS sobre saúde feminina e violência } \\
\text { doméstica contra a mulher. }\end{array}$ & $\begin{array}{l}\text { Em geral, } 38,8 \% \text { relataram ter sido expostas a pelo menos um } \\
\text { tipo de violência durante a gravidez e } 11,5 \% \text { apresentaram } \\
\text { sinais de depressão. O tipo de violência vivida mais comum foi } \\
\text { a violência emocional ( } 30,7 \%) \text {, seguida da violência sexual } \\
(19,0 \%) \text { e da violência fisica (10,0\%). A exposição a pelo } \\
\text { menos um tipo de violência foi o preditor mais forte para DPP, } \\
\text { sendo a violência fisica o mais forte entre as demais violências, } \\
\text { com OR de } 4,42 \text {. }\end{array}$ \\
\hline (12) Ahmad NA, et al. & $\begin{array}{l}\text { Postnatal depression and } \\
\text { intimate partner violence: a } \\
\text { nationwide clinic-based cross- } \\
\text { sectional study in Malaysia. }\end{array}$ & $\begin{array}{l}\text { Estudo transversal de abrangência nacional na Malásia com } \\
5.727 \text { mulheres. A DPP foi avaliada pela EPDS e a VPI foi } \\
\text { avaliada por meio de um Questionário sobre a Saúde da } \\
\text { Mulher e os Eventos de Vida da OMS, validado localmente. }\end{array}$ & $\begin{array}{l}\text { A prevalência de DPP foi de } 4,4 \% \text { e a de VPI foi de } 4,9 \% \text {. Entre } \\
\text { as mulheres desse grupo, } 3,7 \% \text { sofreram violência emocional, } \\
2,6 \% \text { física e } 1,2 \% \text { sexual. As mulheres expostas à VPI tinham } \\
\text { risco } 2,3 \text { vezes maior de desenvolver DPP do que as não } \\
\text { expostas. }\end{array}$ \\
\hline $\begin{array}{l}\text { (13) Lima LS, do } \\
\text { Carmo TOA, Neto } \\
\text { CSB, Pena JLC }\end{array}$ & $\begin{array}{l}\text { Sintomas depressivos em } \\
\text { gestantes e violência por } \\
\text { parceiro intimo: um estudo } \\
\text { transversal. }\end{array}$ & $\begin{array}{l}\text { Estudo piloto transversal entre } 65 \text { gestantes que realizaram } \\
\text { acompanhamento de pré-natal na UBS da Universidade } \\
\text { Federal do Amapá. ADPP foi avaliada por meio da EPDS e a } \\
\text { VPI por meio do Estudo Multi-Países sobre Saúde da Mulher } \\
\text { e Violência Doméstica. }\end{array}$ & $\begin{array}{l}41,5 \% \text { das gestantes apresentaram sintomas depressivos. } \\
\text { Esses sintomas mostraram ter associação significativa em } \\
\text { mulheres que sofreram algum tipo de VPI, com OR de } 6,74 \text {. }\end{array}$ \\
\hline $\begin{array}{l}\text { (14) Miura A, Fujiwara } \\
\text { T. }\end{array}$ & $\begin{array}{l}\text { Intimate partner violence } \\
\text { during pregnancy and } \\
\text { postpartum depression in } \\
\text { Japan: A cross-sectional } \\
\text { study. }\end{array}$ & $\begin{array}{l}\text { Estudo transversal entre } 6.590 \text { mães cadastradas em } \\
\text { programas municipais de saúde para bebês de } 3-4 \text { meses em } \\
\text { Aichi, Japão. A DPP foi avaliada através da EPDS e a } \\
\text { violência doméstica foi avaliada por uma pergunta sobre } \\
\text { violência verbal e outra sobre violência física. }\end{array}$ & $\begin{array}{l}\text { A VPI verbal e física durante a gravidez foi significativamente } \\
\text { associado à DPP. O risco de DPP para mulheres que foram } \\
\text { abusadas verbalmente por seus parceiros durante a gravidez } \\
\text { com uma frequência de "frequentemente" foi } 4,85 \text { vezes maior } \\
\text { em relação às mulheres não abusadas. Já entre mulheres que } \\
\text { foram fisicamente abusadas por seus parceiros durante a } \\
\text { gravidez com uma frequência de "às vezes ou } \\
\text { frequentemente", esse risco foi de } 7,05 \text {. }\end{array}$ \\
\hline $\begin{array}{l}\text { (15) Zhang Y, Zou S, } \\
\text { Cao Y, Zhang Y. }\end{array}$ & $\begin{array}{l}\text { Relationship between } \\
\text { domestic violence and } \\
\text { postnatal depression among } \\
\text { pregnant Chinese women. }\end{array}$ & $\begin{array}{l}\text { Estudo transversal entre } 215 \text { mulheres grávidas selecionadas } \\
\text { com o Abuse Assessment Screen. ADPP foi avaliada por } \\
\text { meio da EPDS. }\end{array}$ & $\begin{array}{l}\text { A prevalência geral de violência doméstica durante a gravidez } \\
\text { foi de } 11,3 \% \text {, e a forma mais comum de violência doméstica foi } \\
\text { o abuso psicológico. As entrevistas de acompanhamento } \\
\text { mostraram uma prevalência maior de DPP entre as mulheres } \\
\text { que sofreram abuso durante a gravidez }(25,1 \%) \text { do que entre } \\
\text { aquelas que não sofreram }(6,0 \%) \text {. }\end{array}$ \\
\hline $\begin{array}{l}\text { (16) Necho M, Belete } \\
\text { A, Zenebe Y. }\end{array}$ & $\begin{array}{l}\text { The association of intimate } \\
\text { partner violence with } \\
\text { postpartum depression in } \\
\text { women during their first month } \\
\text { period of giving delivery in } \\
\text { health centers at Dessie town, } \\
2019 \text {. }\end{array}$ & $\begin{array}{l}\text { Estudo transversal entre } 378 \text { puérperas na clinica de saúde } \\
\text { materno-infantil dos centros de saúde de Dessie, } 4 \text { semanas } \\
\text { após o parto. A DPP foi avaliada por meio da EPDS e a VPI } \\
\text { foi operacionalizada como uma ação abusiva psicológica, } \\
\text { física e sexual imposta às mulheres por seus companheiros. }\end{array}$ & $\begin{array}{l}\text { Mais de um quarto das mulheres entrevistadas (27\%) } \\
\text { apresentaram DPP. A VPI demonstrou associação } \\
\text { estatisticamente significativa com a DPP. A VPI psicológica } \\
\text { apresentou OR de 6,5 enquanto a VPI sexual e física } \\
\text { apresentaram OR de } 3,46 \text { para desenvolvimento de DPP. }\end{array}$ \\
\hline $\begin{array}{l}\text { (17) Hou F, Zhang X, } \\
\text { Cerulli C, He W, Mo Y, } \\
\text { Gong W. }\end{array}$ & $\begin{array}{l}\text { The impact of intimate partner } \\
\text { violence on the trajectory of } \\
\text { perinatal depression: A cohort } \\
\text { study in a Chinese sample. }\end{array}$ & $\begin{array}{l}\text { Estudo de coorte entre } 813 \text { gestantes acompanhadas durante } \\
\text { o período perinatal em Hunan, China. A coleta de dados } \\
\text { ocorreu em sete momentos: quando os participantes foram } \\
\text { aos hospitais para os primeiros exames pré-natais, } 7 \text { dias } \\
\text { após o parto e } 6^{a} \text { semana após o parto. A DPP foi avaliada } \\
\text { por meio da EPDS. Para avaliar a VPI foram adotados } 3 \text { itens } \\
\text { (violência física, violência psicológica e violência sexual) de } \\
\text { vitimização da CTS2S. Esses itens foram incluídos na Escala } \\
\text { de Resiliência Breve (BRS). }\end{array}$ & $\begin{array}{l}\text { Das participantes, } 11,07 \% \text { relataram experiência de VPI nos } \\
\text { últimos } 12 \text { meses. Com relação à violência física, psicológica e } \\
\text { sexual, a prevalência foi de } 4,55 \%, 9,23 \% \text { e } 2,34 \% \text {. A DPP foi } \\
\text { associada à VPI, apresentando um OR de } 3,74 \text {. } \\
\text { Adicionalmente, a vitimização pela VPI está associada com } \\
\text { aumento da gravidade e prognóstico lento dos sintomas de } \\
\text { DPP }\end{array}$ \\
\hline
\end{tabular}




\begin{tabular}{|c|c|c|c|}
\hline Autor & Titulo & Características metodológicas & Principais achados \\
\hline $\begin{array}{l}\text { (18) Finnbogadóttir } \mathrm{H} \text {, } \\
\text { Dykes AK. }\end{array}$ & \begin{tabular}{|l|} 
Increasing prevalence and \\
incidence of domestic violence \\
during the pregnancy and one \\
and a half year postpartum, as \\
well as risk factors: -A \\
longitudinal cohort study in \\
Southern Sweden.
\end{tabular} & $\begin{array}{l}\text { Estudo de coorte longitudinal incluindo } 731 \text { mulheres grávidas } \\
\geq 18 \text { anos de idade na área sudoeste da Suécia. Foram } \\
\text { usados três questionários (QI-III) durante a gravidez e 1-1.5 } \\
\text { anos pós-parto. A DPP foi avaliada por meio da EPDS e a } \\
\text { violência doméstica foi avaliada por meio do Questionário de } \\
\text { Abuso de NorVold (NorAQ), com perguntas sobre violência } \\
\text { psicológica, abuso fisico e sexual e gravidade. }\end{array}$ & $\begin{array}{l}\text { A prevalência de violência doméstica durante a gravidez foi de } \\
2,5 \% \text {. De } 1 \text { a } 1,5 \text { anos após o parto, a prevalência de violência } \\
\text { doméstica aumentou para } 3,3 \% \text {. Assim, a violência doméstica } \\
\text { aumenta com o desenvolvimento da gravidez e pós-parto. A } \\
\text { incidência foi de } 14 \text { por } 1000 \text { mulheres durante a gravidez e } \\
17,2 \text { por } 1000 \text { mulheres pós-parto. Ter vários sintomas de } \\
\text { depressão forneceu um risco } 3,5 \text { vezes maior de violência. }\end{array}$ \\
\hline (19) Reilly, RO. & $\begin{array}{l}\text { Opportunistic domestic } \\
\text { violence screening for } \\
\text { pregnant and post-partum } \\
\text { women by community based } \\
\text { health care providers. }\end{array}$ & $\begin{array}{l}\text { Estudo de métodos mistos com pesquisa e entrevistas } \\
\text { semiestruturadas, com perguntas objetivas e respostas } \\
\text { abertas. A amostra foram profissionais de saúde da } \\
\text { comunidade que prestavam cuidados a mulheres grávidas ou } \\
\text { pós-parto, no Distrito Sanitário Local de Sydney. Os dados } \\
\text { quantitativos das pesquisas foram inseridos no Statistical } \\
\text { Package for the Social Science (SPSS TM Versão 22) e } \\
\text { analisados mediante estatística descritiva. As respostas } \\
\text { abertas foram comparadas e, em seguida, integradas e } \\
\text { apresentadas com os dados quantitativos. }\end{array}$ & $\begin{array}{l}\text { Alguns profissionais de saúde não fizeram a triagem de } \\
\text { violência doméstica. Os fatores que contribuiram para essa } \\
\text { falta de rastreamento incluíram: a falta de reconhecimento de } \\
\text { que isso fazia parte de seu papel; e uma falta de políticas de } \\
\text { triagem de violência doméstica e / ou sistemas de lembretes. } \\
\text { Outras barreiras ao rastreamento da violência doméstica foram } \\
\text { identificadas como falta de tempo, recursos e confiança para } \\
\text { realizar o rastreamento e o encaminhamento das mulheres } \\
\text { quando a violência doméstica foi detectada. }\end{array}$ \\
\hline $\begin{array}{l}\text { (20) Mokhele I, Nattey } \\
\text { C, Jinga N, } \\
\text { Mongwenyana C, Fox } \\
\text { MP, Onoya D. }\end{array}$ & $\begin{array}{l}\text { Prevalence and predictors of } \\
\text { postpartum depression by HIV } \\
\text { status and timing of HIV } \\
\text { diagnosis in Gauteng, South } \\
\text { Africa. }\end{array}$ & $\begin{array}{l}\text { Estudo transversal entre } 1151 \text { mulheres infectadas com HIV-1 } \\
\text { pós-parto (690) e HIV negativas (461) que deram à luz até } 30 \\
\text { dias antes da inscrição no estudo, entrevistadas nas unidades } \\
\text { obstétricas em Gauteng, Africa do Sul. A DPP foi avaliada por } \\
\text { meio da escala CES-D 10. }\end{array}$ & $\begin{array}{l}\text { No geral, um quarto das mulheres tinham DPP. Uma proporção } \\
\text { maior de mulheres HIV negativas experimentaram DPP, sendo } \\
28,0 \% \text { entre HIV negativas e } 23,0 \% \text { entre infectadas por HIV-1. } \\
\text { Entre as mulheres HIV positivas, não houve diferença } \\
\text { significativa entre os recém-diagnosticados com HIV e aqueles } \\
\text { diagnosticados antes da gravidez mais recente. }\end{array}$ \\
\hline $\begin{array}{l}\text { (21) Do TKL, Nguyen } \\
\text { TTH, Pham TTH. }\end{array}$ & $\begin{array}{l}\text { Postpartum Depression and } \\
\text { Risk Factors among } \\
\text { Vietnamese Women. }\end{array}$ & $\begin{array}{l}\text { Estudo transversal entre } 116 \text { mulheres no pós-parto em } \\
\text { Hanói, Vietnã. ADPP foi avaliada por meio da EPDS. As } \\
\text { novas mães também foram solicitadas a relatar informações } \\
\text { sobre dados demográficos, histórico de gravidez e doença, } \\
\text { habilidade interpessoal e características. }\end{array}$ & $\begin{array}{l}\text { A prevalência de DPP durante o primeiro ano após o parto foi } \\
\text { de } 27,6 \% \text {. Mulheres com doenças gestacionais apresentam } \\
\text { maior fator de risco de DPP em comparação com outras } \\
\text { mulheres, assim como mulheres com comunicação / interação } \\
\text { limitada com outras pessoas e insatisfação com sua vida } \\
\text { familiar. Além disso, mães com menor nivel de educação eram } \\
\text { menos propensas a ter DPP, enquanto aquelas que se } \\
\text { tornaram mães pela primeira vez tinham mais de } 2 \text { vezes mais } \\
\text { chances de ter DPP do que aquelas que já tinham filhos. }\end{array}$ \\
\hline $\begin{array}{l}\text { (22) Tolossa T, } \\
\text { Fetensa G, Yilma MT, } \\
\text { Abadiga M, Wakuma } \\
\text { B, Besho M, et al. }\end{array}$ & $\begin{array}{l}\text { Postpartum depression and } \\
\text { associated factors among } \\
\text { postpartum women in } \\
\text { Ethiopia: a systematic review } \\
\text { and meta-analysis, } 2020 \text {. }\end{array}$ & $\begin{array}{l}\text { Revisão sistemática e meta-análise da literatura, com o intuito } \\
\text { de determinar a prevalência de DPP e seus fatores } \\
\text { associados em mulheres pós-parto na Etiópia. Foram } \\
\text { incluidos } 12 \text { artigos na análise final. }\end{array}$ & $\begin{array}{l}\text { A magnitude geral combinada da DPP foi de } 22,89 \% \text { com a } \\
\text { menor }(12,20 \%) \text { e a maior }(33,82 \%) \text { na região das naç̃̃es do } \\
\text { sul. Gravidez não planejada, violência doméstica, falta de apoio } \\
\text { social, história anterior de depressão, perda de filhos e } \\
\text { insatisfação no casamento mostraram associação } \\
\text { estatisticamente significativa com depressão pós-parto. }\end{array}$ \\
\hline $\begin{array}{l}\text { (23) Stewart DE, } \\
\text { Robertson E, Phil M, } \\
\text { Dennis C, Grace SL, } \\
\text { Wallington T. }\end{array}$ & $\begin{array}{l}\text { Postpartum Depression: } \\
\text { Literature review of risk factors } \\
\text { and interventions. }\end{array}$ & $\begin{array}{l}\text { Revisão critica da literatura foi realizado por uma equipe de } \\
\text { pesquisa acadêmica no Programa de Saúde da Mulher da } \\
\text { University Health Network. Todos os artigos relevantes foram } \\
\text { avaliados criticamente e sua qualidade classificados em } \\
\text { niveis de evidência e força de recomendação com base na } \\
\text { metodologia padronizada desenvolvida pela Força-Tarefa } \\
\text { Canadense em Cuidados de Saúde Preventivos. }\end{array}$ & $\begin{array}{l}\text { A DPP afeta aproximadamente } 13 \% \text { das mulheres em um ano } \\
\text { após o parto. Os seguintes fatores de risco são fortes } \\
\text { preditores de DPP: depressão ou ansiedade durante a } \\
\text { gravidez, eventos estressantes da vida recente, problemas } \\
\text { sociais e história prévia de depressão. Preditores moderados } \\
\text { de DPP são estresse pelos cuidados com filhos, baixa } \\
\text { autoestima, neuroticismo materno e temperamento infantil } \\
\text { difícil. Pequenos preditores incluem complicações obstétricas, } \\
\text { atribuições cognitivas negativas, estado civil solteiro, pobre } \\
\text { relacionamento com o parceiro e status socioeconômico } \\
\text { inferior, incluindo renda. }\end{array}$ \\
\hline $\begin{array}{l}\text { (24) Zinga D, Phillips } \\
\text { SD, Born L. }\end{array}$ & $\begin{array}{l}\text { Postpartum depression: We } \\
\text { know the risks, can it be } \\
\text { prevented? }\end{array}$ & $\begin{array}{l}\text { Revisão da literatura atual sobre a etiologia e os fatores de } \\
\text { risco para DPP e as estratégias psicossociais e } \\
\text { farmacológicas que têm sido tentadas no esforço de prevenir } \\
\text { seu aparecimento em mulheres em risco. }\end{array}$ & $\begin{array}{l}\text { A pesquisa aponta para um forte componente biológico } \\
\text { subjacente à sua etiologia. Um monitoramento próximo no } \\
\text { primeiro ano pós-parto, especialmente em mulheres com } \\
\text { história de DPP, é crucial. Algumas perguntas simples durante } \\
\text { as visitas de rotina podem ser eficazes na identificação de } \\
\text { mulheres em risco de DPP, abordando a história psiquiátrica } \\
\text { pessoal e familiar, em particular os transtornos do humor e o } \\
\text { alcoolismo e estabelecer o estado de espírito e o humor atuais } \\
\text { das mulheres. A interrupção abrupta de regimes psicotrópicos } \\
\text { durante a gravidez deve ser evitada para evitar o agravamento } \\
\text { da saúde psicológica. }\end{array}$ \\
\hline $\begin{array}{l}\text { (25) Faisal-Cury A, } \\
\text { Tabb K, Matijasevich } \\
\text { A. }\end{array}$ & $\begin{array}{l}\text { Partner relationship quality } \\
\text { predicts later postpartum } \\
\text { depression independently of } \\
\text { the chronicity of depressive } \\
\text { symptoms. }\end{array}$ & $\begin{array}{l}\text { Estudo prospectivo entre } 294 \text { puérperas de baixa renda } \\
\text { realizada em } 12 \text { Unidades Básicas de Saúde em cinco bairros } \\
\text { da Zona Norte da capital paulista, todas com Estratégia } \\
\text { Saúde da Família implantada. Foi usado um questionário } \\
\text { estruturado e o Patient Health Questionnaire-9 (PHQ-9) para } \\
\text { a variável de desfecho principal e covariáveis. Este estudo } \\
\text { buscou examinar a associação da qualidade do } \\
\text { relacionamento com o parceiro e declínio da vida sexual com } \\
\text { DPP materno em 12-15 meses após o parto. }\end{array}$ & $\begin{array}{l}\text { A prevalência de DPP } 12 \text { a } 15 \text { meses após o parto (DPP tardia) } \\
\text { foi de } 19,1 \% \text {. Uma baixa qualidade de relacionamento com } \\
\text { parceiro apresentou RR de } 1,58 \text { e o declínio da vida sexual } \\
\text { teve um RR de } 1,97 \text {. A DPP tardia é muito comum entre } \\
\text { mulheres de baixa renda e está associada a diferentes } \\
\text { aspectos do relacionamento do casal. Melhorar a qualidade do } \\
\text { relacionamento com o parceiro pode evitar a DPP tardia. }\end{array}$ \\
\hline $\begin{array}{l}\text { (26) Koirala P, } \\
\text { Chuemchit M. }\end{array}$ & $\begin{array}{l}\text { Depression and Domestic } \\
\text { Violence Experiences Among } \\
\text { Asian Women: A Systematic } \\
\text { Review. }\end{array}$ & $\begin{array}{l}\text { Revisão sistemática com metanálise com inclusão de artigos } \\
\text { qualitativos e quantitativos realizados com a população } \\
\text { asiática. Foram incluídos } 38 \text { artigos dos seguintes paises: Irã, } \\
\text { Malásia, Paquistão, Japão, Bangladesh, Nepal, Turquia, } \\
\text { İndia, Vietnã, Israel, China, Tailândia e Timor-Leste. }\end{array}$ & $\begin{array}{l}\text { A prevalência de DPP variou entre } 8,2 \% \text { a } 70 \% \text { e a de violência } \\
\text { doméstica entre } 6,1 \% \text { a } 67,4 \% \text {. Houve } 1,6 \text { a } 7,1 \text { maiores } \\
\text { chances de DPP entre aquelas que sofreram violência } \\
\text { doméstica. A VPI foi o fator mais relevante e de maior risco } \\
\text { para as mulheres que sofreram abuso psicológico (que foi a } \\
\text { forma mais prevalente de VPI), e a violência / intimidação por } \\
\text { outros membros da familia também foi associada ao DPP.A } \\
\text { violência doméstica aumentou o risco de ideação suicida na } \\
\text { DPP. }\end{array}$ \\
\hline $\begin{array}{l}\text { (27) Zhang S, Wang L, } \\
\text { Yang T, Chen L, Qiu } \\
\text { X, Wang T, et al. }\end{array}$ & $\begin{array}{l}\text { Maternal violence experiences } \\
\text { and risk of postpartum } \\
\text { depression: A meta-analysis } \\
\text { of cohort studies. }\end{array}$ & $\begin{array}{l}\text { Revisão sistemática com metanálise para avaliar a relação } \\
\text { entre experiências de violência materna e o risco de } \\
\text { desenvolvimento de DPP. Foram incluidos } 32 \text { artigos na } \\
\text { análise final. }\end{array}$ & $\begin{array}{l}\text { As mulheres que vivenciaram qualquer evento de violência } \\
\text { estavam em maior risco de desenvolver DPP (OR de } 2,04 \text { ). } \\
\text { Além disso, diferentes tipos de eventos de violência, como } \\
\text { violência sexual (OR de } 1,56 \text { ), emocional (OR de } 1,75) \text { e } \\
\text { violência física (OR de } 1,90) \text {, bem como doméstica (OR de } \\
2,05 \text { ) ou violência infantil }(\mathrm{OR} \text { de } 1,59 \text { ) também aumentaram o } \\
\text { risco de desenvolver DPP. }\end{array}$ \\
\hline
\end{tabular}

FONTE: Própria, 2021 
A DPP ocorre entre 4 e 6 semanas após o parto, tornandose reconhecidamente um problema de saúde pública, com significativa morbimortalidade materna e infantil, apresentando prevalência entre $10 \%$, em países de alta renda, e 60\% na população da África Subsaariana (12, 20, 21). As puérperas apresentam sinais de irritabilidade, ansiedade, alterações do humor e do sono, além de prejuízo em sua qualidade de vida (12).

Os resultados revelaram que houve associação entre a prática de VD e DPP, sendo que a VPI foi o tipo mais comumente encontrado. Analisando-se os estudos transversais, a prevalência de DPP encontrada ficou entre $4,4 \%$ e $41,5 \%$, correspondendo ao encontrado na literatura mundial.

A DPP tem natureza multifatorial, com diversos fatores de risco envolvidos, incluindo antecedentes pessoais ou familiares de depressão/doenças psiquiátricas, humor depressivo durante a gestação e ansiedade pré-natal, alcoolismo, falta de suporte social/rede de apoio, assim como baixa qualidade no relacionamento com o parceiro e experiências de violência $(22,23,24,25)$.

Todos os estudos desta revisão apresentaram relação entre algum tipo de violência e o desenvolvimento de DPP. A prevalência de casos de violência encontrada foi, nos estudos transversais, de 4,9\% 12, na Malásia, a 67,4\% (9) na Tanzânia, e nos estudos longitudinais, entre 11,07\% 17, na China, e 44\% (8) na Tunísia. Esses achados são compatíveis com os achados da literatura, que apresentaram uma prevalência de violência doméstica entre $6,1 \%$ e $77,2 \%(26)$.

Mulheres vítimas de violência doméstica possuem reconhecidamente um maior risco de desenvolver a DPP e uma explicação provável seria 0 desequilíbrio neuroendócrino provocado pelos episódios de violência, seja ela física, verbal ou psicológica, por se tratar de eventos traumáticos e estressores (27). Apesar da violência psicológica/social ser a mais prevalente, ela não pareceu estar diretamente associada ao desenvolvimento da DPP, ao contrário da violência física $(9,11,14)$.

Rogathi et al. (2017) trouxeram um dado que é pouco debatido na literatura mundial: a influência da faixa etária no desenvolvimento da DPP. O estudo de coorte, realizado na Tanzânia, identificou que mulheres com idade entre 18 e 24 anos, expostas a episódios de violência física, apresentaram 4 vezes mais chances de desenvolver DPP quando comparadas às faixas etárias superiores.

O estudo transversal realizado por Mokhele et al. (2019) apesar de não estabelecer uma relação entre os episódios de violência e DPP, identificou uma baixa prevalência de DPP em mulheres portadoras de HIV/AIDS, possivelmente em razão do estigma que envolve a doença. Ao contrário, Manongi et al. (2020) identificaram uma forte relação entre experiências de violência emocional e desenvolvimento de DPP em mulheres portadoras de HIV/AIDS na Tanzânia, sendo que essa chance foi de até 3 vezes maior quando comparado às mulheres que não eram portadoras de HIV/AIDS.

O diagnóstico e tratamento adequados da DPP é de fundamental importância para a recuperação da saúde mental materna e o estabelecimento do vínculo mãe-filho. Dentre as principais consequências da DPP nesse período, pode-se ressaltar a diminuição do cuidado com o recém-nascido (24). Um importante achado desta revisão foi o dado apresentado por Tabb et al. (2018), que identificaram o suicídio como uma das principais consequências da DPP.

Neste estudo, realizado no Brasil, a prevalência de DPP foi de $28 \%$ e, dentre essas mulheres, $89 \%$ delas apresentaram algum tipo de ideação suicida.

Quanto ao método diagnóstico utilizado, a Escala de Depressão Pós-Parto de Edimburgo (EPDS) foi a mais utilizada para realizar o diagnóstico de DPP. Entretanto, o estudo transversal realizado por Santos et al. (2007) revelou que esse método, de maneira geral, apresenta uma baixa aplicabilidade para o diagnóstico de DPP. Apesar desse apontamento, evidencia-se a importância da realização de novos estudos para que comparações mais robustas sejam realizadas.

Por fim, salienta-se a importância de profissionais treinados para realizar o rastreamento de casos de violência e, consequentemente, da triagem para o desenvolvimento de DPP, assim como apontado por Reilly et al. (2018), realizado na Austrália. O diagnóstico precoce favorece o tratamento e reversão do quadro, diminuindo o risco de se desenvolver complicações maternas e infantis. Dentre as alternativas preventivas, destacam-se as medidas farmacológicas e não farmacológicas, nas quais podem ser incluídas as abordagens psicoterapêuticas, estratégias educacionais e o aprimoramento do cuidado pré e pós-natal (24).

Além disso, deve ser ressaltada a importância das medidas de prevenção contra a própria violência, visto que esse é um importante fator de risco para o desenvolvimento da DPP. Leis e políticas públicas que assegurem a proteção das mulheres, sobretudo das gestantes e puérperas, é de extrema necessidade para a diminuição do impacto desse fator. Assim, frisa-se a Lei Maria da Penha, Lei no 11.340/2006, no Brasil, que tem como objetivo coibir e prevenir a violência doméstica e familiar contra a mulher (1).

Algumas limitações foram identificadas no presente estudo. Em primeiro lugar, a metodologia utilizada incluiu diversos estudos (coorte, transversal, multicêntricos e revisões) de diferentes nacionalidades, o que dificulta a definição exata da população-alvo e, consequentemente, a comparação com a população geral. Apesar de ser compreendida como uma limitação, ressalta-se a importância de estudos que objetivem análises a nível global.

Em segundo lugar, ainda em relação à metodologia, ressaltase a baixa qualidade das evidências dos estudos investigados. Além disso, foram incluídas revisões de literatura, ou seja, dados secundários foram examinados e os artigos foram 
separados em grupos e lidos de maneira independente por apenas um dos autores, o que compromete o processo de seleção.

Em terceiro lugar, ferramentas heterogêneas foram utilizadas para fazer o diagnóstico de DPP e a constatação da ocorrência da VD, impossibilitando a padronização dos resultados encontrados. Por fim, a escassez de estudos avaliando o impacto da faixa etária, da presença de HIV/AIDS e de outras doenças crônicas comprometem a análise da relação da violência doméstica e do desenvolvimento da DPP nesses grupos.

\section{CONCLUSÃO}

Conclui-se, então, que a DPP impacta negativamente a vida das mulheres e de seus filhos, podendo levar a falta de vínculo e até ao suicídio materno. Assim, foi observada uma maior chance de desenvolver DPP entre mulheres com histórico de VPI ou VD, seja essa violência física, verbal ou psicológica. Em relação às mulheres portadoras de HIV/AIDS, essa relação pode se mostrar alterada. Além disso, foi relatado a influência da DPP com ideações suicidas, o maior risco de desenvolver DPP em mulheres mais jovens, a relação da história depressiva com o desenvolvimento de DPP e que existem barreiras ao rastreamento de VDs por parte dos profissionais de saúde. A fim de minimizar a VD e assim diminuir os riscos da DPP, conforme foi evidenciada a associação por meio do presente estudo, é de extrema importância o desenvolvimento e planejamento de intervenções para aumentar o rastreamento da violência doméstica e a promoção de práticas de encaminhamento apropriadas pelos profissionais de saúde. Estudos futuros devem incluir o impacto da faixa etária, da presença de HIV/AIDS e outras doenças crônicas na relação da violência doméstica e do desenvolvimento da DPP, além de definirem melhor a população-alvo e padronizarem as ferramentas de investigação, a fim de se delinear melhores estratégias de combate para assegurar o bem estar das gestantes.

\section{CONFLITO DE INTERESSE}

Os autores confirmam que não há qualquer potencial conflito de interesse relacionado à pesquisa.

\section{FINANCIAMENTO}

Não houve a utilização de recursos financeiros para a realização do trabalho.

\section{REFERÊNCIAS}

1. Brasil. Lei Maria da Penha: Lei no 11.340, de 7 de agosto de 2006 [Internet]. Brasília: Câmara dos Deputados, Edições Câmara. 2010. 34 p. Disponível em: http://www.onu.org.br/img/2014/09/DUDH.pdf\%0Ahttp://w ww.dudh.org.br/wp-

content/uploads/2014/12/dudh.pdf\%0Ahttp://www.planalto. gov.br/ccivil_03/LEIS/L8069.htm\%0Ahttp://www.scielo.br/s cielo.php?script=sci_arttext\&pid=S0104$40601999000100003 \& \operatorname{lng}=p t \&$ tIng $=p t$

2. Fonseca DH, Ribeiro CG, Leal NSB. Violência Doméstica Contra a Mulher: Realidades E Representações Sociais Domestic Violence Against Women: Realities and Social Representations. Psicol Soc. [Internet]; 2012;24(2):30714. Disponível em: https://doi.org/10.1590/S0102$\underline{71822012000200008}$

3. Cruz EBS, Simões GL, Faisal-Cury A. Rastreamento da depressão pós-parto em mulheres atendidas pelo Programa de Saúde da Família. Rev. Bras. Ginecol. Obstet. [Internet]. 2005 Abril [acesso em 11 Maio 2021] ; 27( 4 ): 181-188. Disponível em: http://www.scielo.br/scielo.php?script=sci_arttext\&pid=S01 00-72032005000400004\&lng=en. https://doi.org/10.1590/S0100-72032005000400004.

4. Amaro H, Fried LE, Cabral H, Zuckerman B. Violence during pregnancy and substance use. Am J Public Health. [Internet] 1990;80(5):575-9. Disponível em: https:/www.ncbi.nlm.nih.gov/pmc/articles/PMC1404633/

5. Stone PW. Popping the (PICO) question in research and evidence-based practice. Appl Nurs Res [Internet] 2002 August;15(3):197-8. Disponível em: https://pubmed.ncbi.nIm.nih.gov/12173172/

6. Gibbs A, Carpenter B, Crankshaw T, Hannass-Hancock J, Smit J, Tomlinson $\mathrm{M}$, et al. Prevalence and factors associated with recent intimate partner violence and relationships between disability and depression in postpartum women in one clinic in eThekwini Municipality, South Africa. PLoS One. [Internet] 2017;12(7):1-12.

7. Tabb KM, Huang $H$, Valdovinos $M$, Toor $R$, Ostler $T$, Vanderwater $\mathrm{E}$, et al. Intimate Partner Violence Is Associated with Suicidality among Low-Income Postpartum Women. J Women's Heal. [Internet] 2018;27(2):171-8.

8. Ghajati B, Bram N, Tajmout A, Ben Romdhane I, Homri W, Labbane R. Domestic Violence and Postpartum Depression: a Study About 25 Tunisian Women. European Psychiatry [Internet]; 2015; 30,131

9. Islam MJ, Broidy L, Baird K, Mazerolle P. Intimate partner violence around the time of pregnancy and postpartum depression: The experience of women of Bangladesh. PLoS One. [Internet] 2017;12(5):e0176211. 
10. Rogathi JJ, Manongi R, Mushi D, Rasch V, Sigalla GN, Gammeltoft T, et al. Postpartum depression among women who have experienced intimate partner violence: A prospective cohort study at Moshi, Tanzania. J Affect Disord [Internet]. 2017;218(Abril):238-45. Disponível em: http://dx.doi.org/10.1016/j.jad.2017.04.063

11. Manongi R, Rogathi J, Sigalla G, Mushi D, Rasch V, Gammeltoft $T$, et al. The Association Between Intimate Partner Violence and Signs of Depression During Pregnancy in Kilimanjaro Region, Northern Tanzania. J Interpers Violence. [Internet] 2020;35(23-24):5797-811.

12. Ahmad NA et al. Postnatal depression and intimate partner violence: a nationwide clinic-based cross-sectional study in Malaysia. BMJ Open [Internet] 8, 20649 (2018).

13. Lima LS, do Carmo TOA, Neto CSB, Pena JLC. Sintomas depressivos em gestantes e violência por parceiro íntimo: um estudo transversal. Enfermería Glob. [Internet];2020; 19, $1-45$.

14. Miura A, Fujiwara T. Intimate partner violence during pregnancy and postpartum depression in Japan: A crosssectional study. Front Public Heal. [Internet] 2017;5(APR):17.

15. Zhang Y, Zou S, Cao Y, Zhang Y. Relationship between domestic violence and postnatal depression among pregnant Chinese women, International Journal of Gynecology \& Obstetrics [Internet], 2012;26-3

16. Necho $M$, Belete $A$, Zenebe $Y$. The association of intimate partner violence with postpartum depression in women during their first month period of giving delivery in health centers at Dessie town, 2019. Ann Gen Psychiatry [Internet];2020; 19, 59 .

17. Hou F, Zhang X, Cerulli C, He W, Mo Y, Gong W. The impact of intimate partner violence on the trajectory of perinatal depression: A cohort study in a Chinese sample. Epidemiol Psychiatr Sci. [Internet] 2020;

18. Finnbogadóttir $H$, Dykes AK. Increasing prevalence and incidence of domestic violence during the pregnancy and one and a half year postpartum, as well as risk factors: -A longitudinal cohort study in Southern Sweden. BMC Pregnancy Childbirth [Internet]. 2016;16(1):1-12. Disponível em: http://dx.doi.org/10.1186/s12884-0161122-6.

19. Reilly, R. O. Opportunistic domestic violence screening for pregnant and post-partum women by community based health care providers. BMC Women's Heal. [Internet]; 2018, $1-8$.
20. Mokhele I, Nattey C, Jinga N, Mongwenyana C, Fox MP, Onoya D. Prevalence and predictors of postpartum depression by HIV status and timing of HIV diagnosis in Gauteng, South Africa. PLoS One. [Internet] 2019;14(4):e0214849.

21. Do TKL, Nguyen TTH, Pham TTH. Postpartum Depression and Risk Factors among Vietnamese Women. Biomed Res Int. [Internet] 2018; 2018:4028913.

22. Tolossa T, Fetensa G, Yilma MT, Abadiga M, Wakuma B, Besho $M$, et al. Postpartum depression and associated factors among postpartum women in Ethiopia: a systematic review and metaanalysis, 2020. Public Health Rev. [Internet] 2020;41:21.

23. Stewart DE, Robertson E, Phil M, Dennis C, Grace SL, Wallington T. Postpartum Depression: Literature review of risk factors and interventions. WHO Publ [Internet]. 2003;(Outubro):289. Disponível em: http://www.who.int/mental_health/prevention/suicide/lit_rev iew postpartum depression.pdf

24. Zinga D, Phillips SD, Born L. Postpartum depression: We know the risks, can it be prevented? Rev Bras Psiquiatr. [Internet] 2005;27(SUPPL. 2):56-64.

25. Faisal-Cury A, Tabb K, Matijasevich A. Partner relationship quality predicts later postpartum depression independently of the chronicity of depressive symptoms. Brazilian J Psychiatry [Internet]. 2021;43(1):12-21.

26. Koirala P, Chuemchit M. Depression and Domestic Violence Experiences Among Asian Women: A Systematic Review. Int J Womens Health [Internet]. 2020;12:21-33.

27. Zhang S, Wang L, Yang T, Chen L, Qiu X, Wang T, et al. Maternal violence experiences and risk of postpartum depression: $\mathrm{A}$ meta-analysis of cohort studies. Eur Psychiatry [Internet]. 2019 Jan;55:90-101.

28. Santos IS, Matijasevich A, Tavares BF, Barros AJD, Botelho IP, Lapolli $C$, et al. Validation of the Edinburgh Postnatal Depression Scale (EPDS) in a sample of mothers from the 2004 Pelotas Birth Cohort Study. Cad Saude Publica. [Internet] 2007;23(11):2577-88. 